\title{
Linking syntactic and semantic arguments in a dependency-based formalism
}

\author{
Christian Korthals and Ralph Debusmann \\ Computational Linguistics \\ Universität des Saarlandes, Geb. 17 \\ Postfach 151150 \\ 66041 Saarbücken, Germany \\ (cnkortha|rade) @coli.uni-sb.de
}

\begin{abstract}
We propose a formal characterization of variation in the syntactic realization of semantic arguments, using hierarchies of syntactic relations and thematic roles, and a mechanism of lexical inheritance to obtain valency frames from individual linking types. We embed the formalization in the new lexicalized, dependency-based grammar formalism of Topological Dependency Grammar (TDG) (Duchier and Debusmann, 2001). We account for arguments that can be alternatively realized as a NP or a PP, and model thematic role alternations. We also treat auxiliary constructions, where the correspondance between syntactic and semantic argumenthood is indirect. ${ }^{1}$
\end{abstract}

\section{Introduction}

This paper deals with the mapping (or linking) of semantic predicate-argument structure to surface syntactic realizations. We present a formal architecture in the framework of a multi-dimensional, heavily lexicalized, efficiently parsable dependency formalism (Duchier and Debusmann, 2001), which uses lexical inheritance as a means to explicitly model syntactic variation. We concentrate on variation between prepositional phrases and nominal phrases which realize verbal arguments, and remedy problems that occur with this kind of variation in recent approaches like the HPSG linking architecture proposed by (Davis, 1998).

Section 2 presents and analyses some of the problematic data we can model, English dative shift, optional complements and thematic role alternations. Section 3 compares the HPSG account with less formal valency or dependency based approaches and comments on the shortcomings, focusing on the treatment of PP complements. We then present a new account in the formal framework of Topologi-

\footnotetext{
${ }^{1}$ The authors wish to thank Denys Duchier and Geert-Jan Kruijff for lots of helpful comments on this paper.
}

cal Dependency Grammar (TDG) by adding a new representational level (thematic structure additionally to ID structure) to the framework in Section 4.1 and introducing the concept of a valency frame in the TDG inheritance lexicon (Sections 4.2 and 4.3). We then show how we use syntactic role hierarchies to account for the data in a linguistically concise way and define admissibility conditions for a TDG derivation. Section 5 contrasts our analysis of the dative shift construction with the analysis of thematic role alternations.

\section{Linguistic Data}

Insights from corpus studies (e.g. the NEGRA treebank for German (Skut et al., 1998), or the material annotated in the Framenet (Baker et al., 1998) project on the basis of The Bank of English (Cobuild, 2001) show that the syntactic patterns specific verbs occur with vary stongly. Not only do we observe different patterns for different verbs, but also alternative patterns with the same verbs. (1) to (6) illustrate the well-known dative shift (Levin, 1993):45 phenomenon, which occurs with a restricted class of verbs only. While the distinction between (2) and (4) can be explained in terms of lexical semantics, even semantically closely related verbs as English give and deliver can differ in their syntactic behaviour, as the contrast between (1) and (5) shows.
(1) [The postman] gave [him] [ a package].
(2) [The postman] gave [a package] [to him].
(3) [The postman] charged [him] [5 Euros].
(4) *[The postman] charged [5 Euros] [to him].
(5) *[The postman] delivered [him $]$ [ a package].
(6) [The postman] delivered [a package] [to him]. 
In contrast to (Davis, 1998):3:56² for instance, we do not assume a difference in meaning between (1) and (2). ${ }^{3}$ Therefore, in order to compute a semantics from this data without spurious ambiguity, we must be able to express the semantic generalisation that to him and him realize the same semantic argument. It is useful to employ thematic roles in the lexicon and in grammatical descriptions for this purpose. ${ }^{4}$ See e.g. (Tarvainen, 1987), (Helbig, 1989), (Sgall et al., 1986) or the Framenet project (Baker et al., 1998) for different application-oriented sets of thematic roles (or "frame elements"). For discussion see e.g. (Helbig, 1995) or (Kruijff, 2001), for criticism see (Dowty, 1991). We can also use thematic roles to structure verbs into an ontology, as e.g. attempted by (Helbig, 1995), (Davis, 1998) or (Baker et al., 1998) in order to make semantic predictions for syntactic valency patterns. For instance, it is a regularity in English that verbs of charging do not show the dative shift (Levin, 1993), while verbs of change of possession sometimes do.

Now consider the set of German examples in (7) to (11), which all roughly express the proposition Peter robs her of her money. All of the patterns are attested in the NEGRA corpus (Skut et al., 1998), but (10) cannot be found.

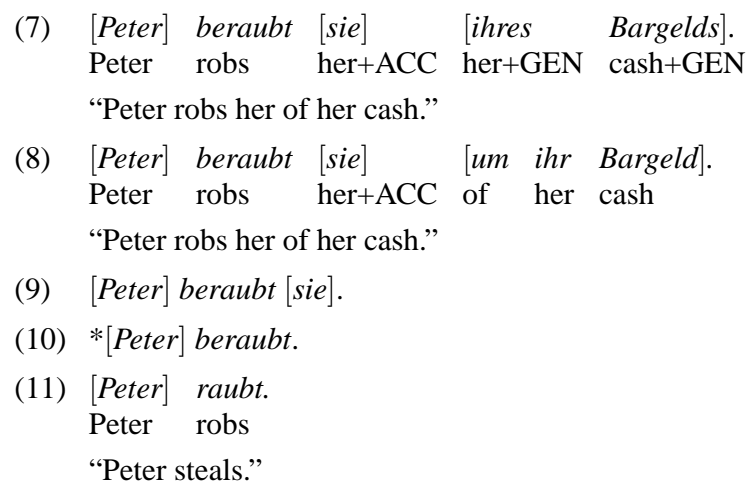

The data illustrates that it can be a lexical property of verbs to allow or prohibit omission of their

\footnotetext{
${ }^{2} \mathrm{We}$ cite the chapter and the page number of the online postscript version at http://www-csli.stanford.edu/ tdavis/

${ }^{3}$ We expect English give to have at least two separate meanings (a) cause someone to physically have something and (b) to cause someone trouble, pain, etc. with different lexical entries, following established lexicographic practice. While the lexical entry for meaning (b) will exhibit the syntactic pattern illustrated by (1) only (*To give headache to someone), the entry for meaning (a) exhibits both the patterns in (1) and (2).

${ }^{4}$ Note that we do not commit ourselves to a specific set of thematic roles in this paper.
}

complements (Levin, 1993):33, (Helbig, 1995):99. Therefore, we will analyse syntactic arguments in terms of optionality and obligatoriness. Note that this distinction is not predictable from the thematic roles realized by the syntactic elements (e.g. by distinguishing inner and outer roles in (Sgall et al., 1986) and (Tarvainen, 1987)) nor by the syntactic form or even function of the syntactic elements. Neither is the distinction between obligatory and optional elements the same as the complement/adjunct distinction.

We analyse (1) to (6) as alternative realizations of a thematic role, because one semantic argument (the PATIENT) can either be realized as indirect object or PP, while the THEME is always realized as a direct object NP. Compare this data to alternations as in (12) and (13). Here, additionally, one syntactic function (direct object) is open for either of two thematic roles (Levin, 1993).

\section{(12) $[\mathrm{He}]$ cleared [the dirt $][$ from the table $]$.}

(13) $[\mathrm{He}]$ cleared $[$ the table $]$ [of the dirt $]$.

We will show in Section 4 how we can account for the data illustrated in this section in a lexicalized dependency grammar formalism and show that the linguistic view taken above helps to reduce redundancy in the lexicon.

\section{Alternative approaches}

The approach taken in this paper formalizes notions that have only been informally employed in dependency grammar. (Helbig, 1995):167 defines valency frames on a formal syntactic and functional syntactic level, a thematic role level and a logical level in his 6-level-model, but only informally and for the purpose of learners' dictionaries. There is a long tradition in German lexicography which has produced a number of valency dictionaries (e.g. (Helbig and Schenkel, 1975), (Fischer, 1997), (Engel and Schumacher, 1976)). The syntactic analyses in these dictionaries are compatible with our model, but they do not provide a thematic role level.

(Melcuk, 1988) characterizes valency frames in a similar fashion (94), but uses informal additions in natural language to constrain the possible patterns. Also (Melcuk, 1988) assumes different levels of representation. A shortcoming of the syntactic level in (Melcuk, 1988) is, though, that his syntactic classes are dependent on the specific lexical item, and therefore problematic to define. The approach 
we will take resembles LFG (Kaplan and Bresnan, 1982) (Bresnan, 2001) in that it assumes syntactic relations.

(Davis, 1998) has recently proposed a linking theory in the formal framework of HPSG. He separates syntax and semantics by postulating thematic roles under the CONTENT feature of his HPSG architecture (Pollard and Sag, 1994), and syntactic characterizations of the arguments under CATEGORY|ARG-ST and CATEGORY|SUBCAT. He has separate hierarchies of syntactic patterns (intrans, trans, ditrans, 5:32) and semantic classes (subtypes of RELATION, 5:72). These hierarchies interact by a set of linking constraints and yield a hierarchy of predicators $(5: 41)$, which specifies possible linkings of thematic roles to syntactic arguments. While (Helbig, 1995) obviously employs a large role set, (Davis, 1998) has only 6 roles, and moves thematic roles further down into semantics than we assume by postulating them on an event level, which "effectively amounts to a limited amount of semantic decomposition" (5:39). The shortcoming of the model is that the syntactic patterns assumed are very sparse indeed with only three transitivity classes. Due to this, semantic predictions can be made only for NP-complements, while PPs must be treated by a separate mechanism ("content sharing account"). Thus, there is no specific prediction for the prepositional complement in English dative shift constructions. The advantage of Davis's model, in contrast, is the lexical inheritance architecture which is a formal means to capture generalizations.

\section{Formalization}

We formalize our idea of linking and valency frames as an extension of the new lexicalized, dependencybased grammar formalism of Topological Dependency Grammar (TDG) (Duchier and Debusmann, 2001), (Debusmann, 2001). So far, TDG is only concerned with syntax: every TDG analysis consists of an unordered dependency tree (ID tree) and an ordered and projective topology tree (LP tree). We only describe a subset of the full TDG grammar formalism (e.g. completely ignoring any issues concerning word order) and extend it with the notion of a thematic graph (TH graph). We call the version of TDG described in this paper $\mathrm{TDG}^{\mathrm{TH}}$.

\subsection{Thematic graphs}

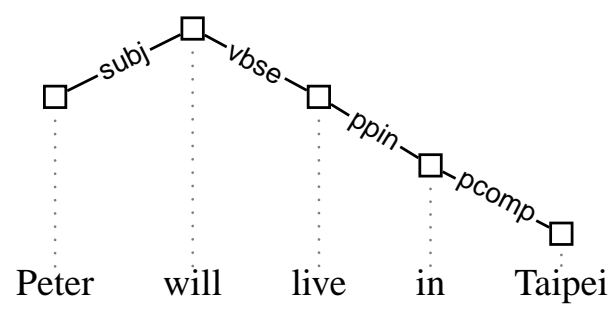

What is a "thematic graph"? We illustrate this notion by an example. (14) is an ID tree analysis for the sentence Peter will live in Taipei: We show the corresponding TH graph in (15). Here, Peter is the patient of will_live and in_Taipei the locative. Note that we collapsed the auxiliary will and its verbal complement live into a single node, and also the PP in_Taipei:

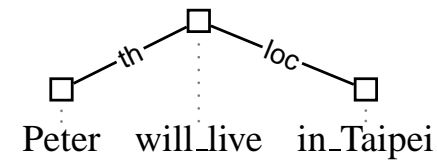

\subsection{The lexicon}

This section deals with the $\mathrm{TDG}^{\mathrm{TH}}$-lexicon. We assume a finite set of syntactic roles $\mathcal{R}$ and a finite set of thematic roles $\mathcal{T}$. We write $\rho$ for a syntactic role in $\mathcal{R}$ and $\theta$ for a thematic role in $\mathcal{T} . \Pi=\{!, ?\}$ is the set of optionality flags $\pi$. $\mathcal{A}=\left\{\mathrm{val}_{\mathrm{ID}}, \mathrm{val}_{\mathrm{TH}}\right.$, link $\}$ is the set of lexical features $\alpha$, and $\mathcal{E}$ the set of lexical entries $e$, having the following signature: ${ }^{5}$

$$
\left[\begin{array}{rll}
\text { val }_{\text {ID }} & : & 2^{\mathcal{R} \times \Pi} \\
\text { val }_{\mathrm{TH}} & : & 2^{\mathcal{T} \times \Pi} \\
\text { link } & : & 2^{\mathcal{T} \times \mathcal{R}}
\end{array}\right]
$$

$\mathcal{E}$ is a lattice of $\mathrm{TDG}^{\mathrm{TH}}$-lexicon entries; lexical entries either correspond to words or to lexical types which can be inherited (see below).

The value of feature val ${ }_{\text {ID }}$ is a set of pairs $(\rho, \pi)$ of syntactic roles and an optionality flag modeling the concept of syntactic valency. The value of $\mathrm{val}_{\mathrm{TH}}$ a set of pairs $(\theta, \pi)$ of thematic roles and an optionality flag (thematic valency). For convenience, we write $\rho \pi$ for $(\rho, \pi)$, and $\theta \pi$ for $(\theta, \pi)$. The value of link is a set of pairs $(\theta, \rho)$ of thematic and syntactic roles, expressing the mapping between them. We call a pair in this set a linking.

\footnotetext{
${ }^{5}$ We write $\alpha(e)$ to denote the value of feature $\alpha$ at lexical entry $e$.
} 


$$
\text { eat }:\left[\begin{array}{rll}
\mathrm{val}_{\mathrm{ID}} & : & \{\text { subj!, objd? }\} \\
\mathrm{val}_{\mathrm{TH}} & : & \{\text { ag!,th?\}} \\
\text { link } & : & \{(\text { ag, subj }), \\
& & (\text { th, objd })\}
\end{array}\right]
$$

As an example, (16) is a lexical entry for finite eat: eat has an obligatory subject (subj) and an optional direct object (objd) in its syntactic valency. Its thematic valency contains an obligatory AGENT and an optional THEME. The link-feature defines two linkings: one links the AGENT to the subject and the THEME to the direct object.

\subsection{Lexical inheritance}

We introduce a mechanism of lexical inheritance. We write $e=e_{1} \sqcap \ldots \sqcap e_{n}$ for 'lexical entry $e$ inherits from lexical entries $e_{1}, \ldots, e_{n}$ ', and define inheritance as the set union of the individual features' values:

$$
\begin{aligned}
& e_{1} \sqcap \ldots \sqcap e_{n}= \\
& {\left[\begin{array}{rl}
\operatorname{val}_{\mathrm{ID}}: & \operatorname{val}_{\mathrm{ID}}\left(e_{1}\right) \cup \ldots \cup \operatorname{val}_{\mathrm{ID}}\left(e_{n}\right) \\
\operatorname{val}_{\mathrm{TH}}: & \operatorname{val}_{\mathrm{TH}}\left(e_{1}\right) \cup \ldots \cup \operatorname{val}_{\mathrm{TH}}\left(e_{n}\right) \\
\operatorname{link}: & \operatorname{link}\left(e_{1}\right) \cup \ldots \cup \operatorname{link}\left(e_{n}\right)
\end{array}\right]}
\end{aligned}
$$

We can now use lexical inheritance to model our notion of valency frames. We introduce the notion of a linking type as a lexical entry that does not specify any other lexical attributes besides $\mathrm{val}_{\mathrm{ID}}$, $\mathrm{val}_{\mathrm{TH}}$ and link. Such linking types specify a partial valency frame from which we can build complete valency frames by inheritance. For instance, consider the following two linking types:

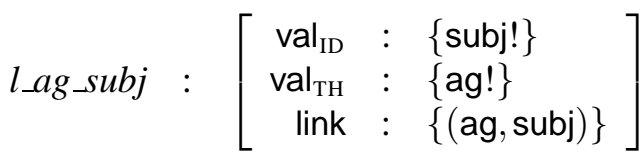

$$
\begin{aligned}
& \text { l_th_objd : }\left[\begin{array}{rll}
\mathrm{val}_{\mathrm{ID}} & :\{\text { objd? }\} \\
\mathrm{val}_{\mathrm{TH}}: & \{\text { th? }\} \\
\text { link } & :\{(\text { th,objd })\}
\end{array}\right]
\end{aligned}
$$

The linking type $l_{-} a g$ subj maps the agent to the subject, and $l_{-}$th_objd the theme to the direct object. Out of the two, we can construct our lexical entry for eat by lexical inheritance:

$$
\text { eat }=\text { l_ag_subj } \sqcap \text { l_th_objd }
$$

which amounts precisely to the lexical entry displayed in (16) above. We call the values of the three features val $\mathrm{ID}_{\mathrm{ID}}$, val $\mathrm{TH}_{\mathrm{TH}}$ and link in a lexical entry obtained by inheriting from linking types a valency frame.

\subsection{Role hierarchies}

We arrange the set $\mathcal{R}$ of syntactic roles in a role hierarchy modeled as a meet semi-lattice. Here is an example cut-out of the role hierarchy:

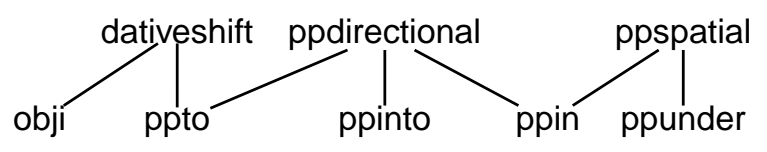

We write $\rho \sqsubseteq \rho^{\prime}$ for $\rho$ is a specialization of $\rho^{\prime}$ (i.e. $\rho$ is below $\rho^{\prime}$ in the hierarchy).

We employ the role hierarchy to model alternative realizations in the sense of section 2: e.g. using the hierarchy above, dativeshift can be realized as either obji or ppto but not by either ppdirectional, ppinto or ppin. Note that certain roles (ppto, ppinto, etc.) will be realized by only two lexical entries, viz. the prepositions to and into respectively, while other roles like subj, obji or objd can be realized by a large set of lexical entries.

In the same fashion, we arrange the set $\mathcal{T}$ of thematic roles in a role hierarchy, but in this article we keep this hierarchy completely flat.

Lexical entry constraint. To forbid that different thematic roles are mapped to the same syntactic role realization, we add a condition for well-formed lexical entries: for every lexical entry $e$, the value of its link-feature, $\operatorname{link}(e)=\left\{\left(\theta_{1}, \rho_{1}\right), \ldots\left(\theta_{n}, \rho_{n}\right)\right\}$ must not include two syntactic roles $\rho_{i}, \rho_{j}(1 \leq i \neq j \leq n)$ such that $\rho_{i} \sqsubseteq \rho_{j}$.

\subsection{TDG $^{\mathrm{TH}}$ analyses}

We can now define a $\mathrm{TDG}^{\mathrm{TH}}$-analysis as a tuple $\left(V, E_{\mathrm{ID}}, \varepsilon, \equiv, E_{\mathrm{TH}}\right)$. It consists of the finite set $V$ of nodes $w$, the finite set $E_{\mathrm{ID}}$ of ID edges $E_{\mathrm{ID}} \subseteq$ $V \times V \times \mathcal{R}$, where $\mathcal{R}$ is the set of syntactic roles, and the lexical assignment function $\varepsilon: V \rightarrow \mathcal{E}$ assigning lexical entries to nodes. We write $w_{1}-\rho \rightarrow{ }_{\mathrm{ID}} w_{2}$ for an ID edge from node $w_{1}$ to node $w_{2}$ labeled with syntactic role $\rho$.

Collapsing nodes. As in the example ID tree and $\mathrm{TH}$ graph-analyses in (14) and (15) above, we would like to be able to collapse sets of nodes in the ID tree into single nodes in the TH graph. We introduce a collapsing principle into the grammar formalism, according to which the node of an auxiliary verb, a preposition or a determiner will collapse with its daughter.

To capture this idea, we posit an equivalence relation $\equiv$, and write $V / \equiv$ for the set of equivalence 
classes over $V$. An equivalence class directly corresponds to a "collapsed node" in the TH graph. $E_{\mathrm{TH}} \subseteq(V / \equiv) \times(V / \equiv) \times \mathcal{T}$ is the finite set of of TH edges, and we write $\bar{w}$ for the equivalence class containing $w . \overline{w_{1}}-\theta \rightarrow_{\mathrm{TH}} \overline{w_{2}}$ is a TH edge from node $\overline{w_{1}}$ to node $\overline{w_{2}}$ labeled with $\theta$.

When we collapse nodes, we also collapse their lexical entries: the value of the lexical feature $\alpha$ of a collapsed node $\overline{w_{1}}=\left\{w_{1}, \ldots, w_{n}\right\}$ is the set union of the values assigned to the individual nodes:

$$
\alpha\left(\overline{w_{1}}\right)=\alpha\left(w_{1}\right) \cup \ldots \cup \alpha\left(w_{n}\right)
$$

In the example $\mathrm{TH}$ graph in (15) above, the two nodes will and live are collapsed into the equivalence class will_live. We assume that will is mapped to the following lexical entry:

$$
\text { will : }\left[\begin{array}{rll}
\text { val }_{\mathrm{ID}} & : & \{\text { subj!, vbse! } \\
\text { val }_{\mathrm{TH}} & : & \{\} \\
\text { link } & : & \{\}
\end{array}\right]
$$

Here, we use the independent definition of the val ${ }_{I D}$, $\mathrm{val}_{\mathrm{TH}}$ and link features in order to express that function words like auxiliaries or prepositions realize syntactic arguments which are semantically dependent on different lexical items. This also allows for an elegant treatment of semantically void syntactic arguments as fake-reflexives and non-referential it. ${ }^{6}$

Infinitive live has this lexical entry:

$$
\text { live : }\left[\begin{array}{rll}
\operatorname{val}_{\mathrm{ID}} & : & \{\text { ppspatial! }\} \\
\mathrm{val}_{\mathrm{TH}}: & \{\text { ag!,loc! }\} \\
\text { link }: & \text { (ag, subj) } \\
& & \text { (loc, ppspatial) }\}
\end{array}\right]
$$

When the two nodes collapse into one (will_live), these are the values of $\mathrm{val}_{\mathrm{TH}}$ and link: ${ }^{7}$

$$
\begin{aligned}
\operatorname{val}_{\mathrm{TH}}(\text { will_live }) & =\{\text { ag! }, \text { loc! }\} \\
\text { link }(\text { will_live }) & =\{(\mathrm{ag}, \text { subj }),(\text { loc }, \text { ppspatial })\}
\end{aligned}
$$

Valency constraints. The well-formedness conditions of a $\mathrm{TDG}^{\mathrm{TH}}$-analysis are stated in terms of lexicalized constraints of syntactic valency, thematic valency and linking. The syntactic valency

\footnotetext{
${ }^{6}$ Fake-reflexives occur in German and are reflexive pronouns that do not have any semantic content, as e.g. the German verb sich fürchten (be afraid, sich is the reflexive pronoun). The linking type for a fake reflexive will only specify val $_{\mathrm{ID}}$ : reflpronoun, but empty val $\mathrm{TH}_{\mathrm{TH}}$ and link.

${ }^{7}$ We omit those features that are not relevant to the $\mathrm{TH}$ graph according to its well-formedness conditions. In particular, the value of the val ${ }_{\mathrm{ID}}$ feature is not relevant to the $\mathrm{TH}$ graph.
}

constraint restricts the number of outgoing edges of each node in the ID tree: if $(\rho, !) \in \operatorname{val}_{\mathrm{ID}}(w)$, then $w$ must have precisely one outgoing edge labeled with $\rho^{\prime} \sqsubseteq \rho$, at most one if $(\rho, ?) \in \operatorname{val}_{\mathrm{ID}}(w)$, and none otherwise. Thus, $(\rho, !)$ stands for an obligatory $\rho$ complement of $w$ and $(\rho, ?)$ for an optional one. The thematic valency constraint is defined analogously.

Linking constraint. An edge in the TH graph is only licensed if it satisfies the lexicalized linking constraint. It states that an edge $\overline{w_{1}}-\theta \rightarrow_{\mathrm{TH}} \overline{w_{2}}$ in the $\mathrm{TH}$ graph is licensed only if there is a linking $\left(\theta^{\prime}, \rho^{\prime}\right) \in \operatorname{link}\left(\overline{w_{1}}\right)$ and an edge $w_{1}^{\prime}-\rho \rightarrow{ }_{\mathrm{ID}} w_{2}^{\prime}$ in the ID tree such that ${\overline{w_{1}}}^{\prime}={\overline{w_{1}}},{\overline{w_{2}}}^{\prime}=\overline{w_{2}}, \theta \sqsubseteq \theta^{\prime}$ and $\rho \sqsubseteq \rho^{\prime}$.

Consider the example ID tree and TH graph analyses in (14) and (15) above. The edge will_live $-\mathrm{loc} \rightarrow \rightarrow_{\mathrm{TH}}$ in_Taipei is mandated by the thematic valency of will_live, but it must also be licensed by the linking principle: indeed there is a linking (loc, ppspatial) in link(will_live) and an ID edge live $-\mathrm{ppin} \rightarrow{ }_{\mathrm{ID}}$ in such that $\overline{\text { live }}=$ will_live and $\overline{i n}=$ in_Taipei, and loc $\sqsubseteq$ loc and ppin $\sqsubseteq$ ppspatial.

\section{Application}

This section describes the linguistic examples from section 2 within the framework developed above.

We define a linking type for the English dative shift construction as follows, realizing our notion of an alternative realization from section 2 .

$$
\begin{aligned}
& \text { l_pat_dativeshift: } \\
& {\left[\begin{array}{rl}
\operatorname{val}_{\mathrm{ID}}: & \text { dativeshift! }\} \\
\operatorname{val}_{\mathrm{TH}}: & \{\text { pat! }\} \\
\text { link } & :\{(\text { pat, dativeshift })\}
\end{array}\right]}
\end{aligned}
$$

As can be seen from the role hierarchy in (20), the syntactic role dativeshift can either be realized as obji or ppto. This linking type will be inherited by all English verbs that exhibit the dative-shift alternative realization. For instance, it is inherited by the lexical entry for the English verb give (cf. examples (1) and (2)). Additionally, give inherits from the linking type $l_{-a g}$ subj defined in (17). $l \_a g$ subj will be shared among a large set of other verbs which realize their agents as subjects, thus reducing redundancy in the lexicon.

$$
\begin{aligned}
\text { gives }= & \text { l_ag_subj } \sqcap \\
& \text { lpat_dativeshift } \sqcap \\
& \text { l_th_objd }
\end{aligned}
$$


The lexicon entry for deliver ((5) and (6)) will differ from the one for give by inheriting from

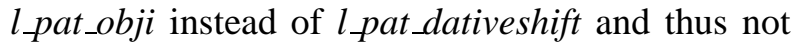
allow for a dative shift.

For the German data in examples (7) to (11) we define a syntactic role hierarchy in the same fashion, where genitive and ppum are below a role ppumgen. Then, the lexical entry for berauben inherits from a linking type $l \_t h e m e$ ppumgen.

In contrast to alternative realizations, alternations as in examples (12) and (13) realize two different thematic roles (pat and th) as the same syntactic role (objd). By the lexical entry constraint in section 4.4, there cannot be a single lexical entry for both alternants of clear. We therefore model finite forms of clear by two separate valency frames (we skip the definitions of some of the linking types).

$$
\begin{aligned}
& \text { clears }=\text { l_ag_subj } \sqcap \\
& \text { l_pat_objd } \sqcap \\
& \text { l_th_ppof } \\
& \text { clears }=\quad l_{\_} \text {ag_subj } \sqcap \\
& \text { l_th_objd } \sqcap \\
& \text { l_pat_ppfrom }
\end{aligned}
$$

\section{Conclusion}

We proposed an architecture that abstractly captures reocurring patterns in the way in which different lexical items syntactically realize their semantic arguments. We focused on interchangeability between prepositional phrases and nominal phrases. We therefore defined a hierarchy of thematic roles, and a separate hierarchy of syntactic functions, clearly separating between syntax and semantics.

We enriched the framework of Topological Dependency Grammar (Duchier and Debusmann, 2001) (TDG) with a third level of representation, thematic structure, and defined well formedness conditions on the thematic structure and on the relation between thematic structure $(\mathrm{TH})$ and syntactic dominance (ID) structure. This enabled us to present a formal definition of the concept of multilevel valency frames. We demonstrated how such valency frames can be assembled using the lexical inheritance model of TDG, in order to reduce lexical redundancy. We also proposed a treatment of auxiliary constructions using a notion of node collapsing.
We applied our formalism to English dative shift constructions, variation between NP and PP in German, optional complements, semantically empty elements, and thematic role alternations.

Our approach makes weaker theoretical predictions about "alternations" as opposed to "alternative realizations" than the approach in (Davis, 1998), but is more powerful in the treatment of PPs. This is partly due to the choice of an underlying dependency formalism, because dependency structures lead to concise and semantically flat parse trees. Our approach is data-driven in the sense that a wider range of syntactic patterns can be mapped to semantic arguments. Thus, it lends itself easy to techniques of automatically acquiring lexica from syntactically and semantically annotated corpora, which start developing at present ((Baker et al., 1998), (Skut et al., 1998)).

It is possible to include a mechanism that makes stronger predictions about alternations of the clear type, or about passivization. Another phenomenon we would like to tackle in this framework are raising and control constructions. We think we can use a concept similar to node collapsing for them.

\section{References}

Collin F. Baker, Charles J. Fillmore, and John B. Lowe. 1998. The Berkeley FrameNet project. In Christian Boitet and Pete Whitelock, editors, 36th ACL and 17th ICCL Proceedings, pages 8690, San Francisco, California. Morgan Kaufmann Publishers.

Joan Bresnan. 2001. Lexical Functional Syntax. Blackwell.

Cobuild. 2001. The bank of english. http://titania.cobuild.collins.co.uk/ boe_info.html.

Tony Davis. 1998. Linking as constraints in the hierarchical lexicon. Chicago UP.

Ralph Debusmann. 2001. A declarative grammar formalism for dependency grammar. Master's thesis, University of the Saarland.

David Dowty. 1991. Thematic proto-roles and argument selection. Language, 67(547-619).

Denys Duchier and Ralph Debusmann. 2001. Topological dependency trees: A constraintbased account of linear precedence. In ACL 2001 Proceedings, Toulouse, France.

Ulrich Engel and Helmut Schumacher. 1976. Kleines Valenzlexikon deutscher Verben. Forschungsbericht IDS Mannheim. 
Klaus Fischer. 1997. German-English Verb Valency. Narr.

Gerhard Helbig and Wolfgang Schenkel. 1975. Wörterbuch zur Valenz und Distribution deutscher Verben. VEB Bibliographisches Institut.

Gerhard Helbig. 1989. Deutsche Grammatik. VEB Enzyklopädie.

Gerhard Helbig. 1995. Probleme der Valenz- und Kasustheorie. Narr.

Ron Kaplan and Joan Bresnan. 1982. Lexical functional grammar: A formal system for grammatical representation. In The mental representation of grammatical relations. MIT Press.

Geert-Jan Kruijff. 2001. A categorial-modal logical architecture of informativity. Ph.D. thesis, Charles University, Prague.

Beth Levin. 1993. English verb classes and alternations. Chicago UP.

Igor Melcuk. 1988. Dependency syntax: Theory and practice. Albany: State Univ of NY.

Carl Pollard and Ivan A. Sag. 1994. Head Driven Phrase Structure Grammar. Chicago UP.

Petr Sgall, Eva Hajicova, and Jarmila Panenova. 1986. The Meaning of the Sentence in its Semantic and Pragmatic Aspects. Reidel.

Wojciech Skut, Thorsten Brants, Brigitte Krenn, and Hans Uszkoreit. 1998. A linguistically interpreted corpus of German newspaper text. In Proceedings of the ESSLLI Workshop on Recent Advances in Corpus Annotation.

Kalevi Tarvainen. 1987. Cases in the framework of dependency grammar. In Concepts of Case. Narr. 